\title{
The possible role of hypoxia in the formation of axonal bulbs
}

\author{
B Kaur, G N Rutty, W R Timperley
}

Department of

Forensic Pathology, University of Sheffield, Medico-Legal Centre, Watery Street, B Kaur

G N Rutty

Department of Neuropathology, Royal Hallamshire Hospital, Sheffield S10 2JF, UK W R Timperley

Correspondence to: Dr Rutty.

email:

G.N.Rutty@sheffield.ac.uk

Accepted for publication 14 September 1998 Sheffield S3 7ES, UK

Abstract

Aims-To assess the possible role of hypoxia in the formation of axonal bulbs. Methods-Study material comprised sections from 28 brains showing evidence of cerebral hypoxia with no history of head injury, four with a history of head trauma but no evidence of hypoxic change, eight with a history of head trauma and hypoxic change, and four from control brains originally described as "diffuse axonal injury." These were subjected to microwave antigen retrieval and immunohistochemistry using monoclonal antibodies to $\beta$ amyloid precursor protein ( $\beta$ APP), glial fibrillary acid protein (GFAP), and CD68PGM1.

Results-Positive staining for $\beta A P P$ was seen in all four controls, all four cases of head injury only, seven of eight cases of

Table 1 Brain histology, immunohistochemical results, brain weights, and number of blocks examined

\begin{tabular}{|c|c|c|c|c|c|c|}
\hline Case & Brain histology & $\beta A P P$ & $G F A P$ & $C D 68$ & Brain weight ( $g$ ) & Number of blocks \\
\hline 1 & DAI & ++ & ++ & +++ & 1368 & 2 \\
\hline 2 & DAI & +++ & ++ & - & 1031 & 3 \\
\hline 3 & DAI & +++ & ++ & +++ & 1500 & 4 \\
\hline 4 & $\mathrm{DAI}$ & +++ & +++ & +++ & 1950 & 2 \\
\hline 5 & $\mathrm{HI}$ & +++ & ++ & +++ & 1510 & 2 \\
\hline 6 & $\mathrm{HI}$ & +++ & ++ & ++ & - & 1 \\
\hline 7 & $\mathrm{HI}$ & ++ & ++ & ++ & 1482 & 1 \\
\hline 8 & $\mathrm{HI}$ & + & +++ & +++ & 1110 & 5 \\
\hline 9 & $\mathrm{HI} \& \mathrm{H}$ & + & ++ & + & 1290 & 3 \\
\hline 10 & $\mathrm{HI} \& \mathrm{H}$ & ++ & ++ & +++ & 1660 & 6 \\
\hline 11 & $\mathrm{HI} \& \mathrm{H}$ & + & + & - & 1270 & 3 \\
\hline 12 & $\mathrm{HI} \& \mathrm{H}$ & + & + & + & 1660 & 2 \\
\hline 13 & HI\&H & + & + & + & 1650 & 5 \\
\hline 14 & $\mathrm{HI} \& \mathrm{H}$ & ++ & - & + & 480 & 3 \\
\hline 15 & $\mathrm{HI} \& \mathrm{H}$ & + & - & - & 1330 & 2 \\
\hline 16 & HI\&H & - & - & - & 1360 & 13 \\
\hline 17 & $\mathrm{H}$ & +++ & +++ & +++ & 1367 & 19 \\
\hline 18 & $\mathrm{H}$ & +++ & +++ & +++ & 680 & 4 \\
\hline 19 & $\mathrm{H}$ & +++ & ++ & ++ & 1320 & 4 \\
\hline 20 & $\mathrm{H}$ & ++ & ++ & ++ & $507(1 / 2)$ & 5 \\
\hline 21 & $\mathrm{H}$ & +++ & ++ & ++ & 1440 & 4 \\
\hline 22 & $\mathrm{H}$ & ++ & - & ++ & 1500 & 4 \\
\hline 23 & $\mathrm{H}$ & +++ & ++ & +++ & 1370 & 5 \\
\hline 24 & $\mathrm{H}$ & + & +++ & ++ & 600 & 3 \\
\hline 25 & $\mathrm{H}$ & +++ & ++ & ++ & 1700 & 4 \\
\hline 26 & $\mathrm{H}$ & +++ & + & ++ & 1525 & 4 \\
\hline 27 & $\mathrm{H}$ & + & +++ & + & 1510 & 3 \\
\hline 28 & $\mathrm{H}$ & ++ & ++ & + & 1470 & 4 \\
\hline 29 & $\mathrm{H}$ & - & - & - & 1060 & 1 \\
\hline 30 & $\mathrm{H}$ & - & - & - & 1600 & 3 \\
\hline 31 & $\mathrm{H}$ & - & - & - & 1120 & 6 \\
\hline 32 & $\mathrm{H}$ & - & - & - & 1320 & 1 \\
\hline 33 & $\mathrm{H}$ & - & - & - & 568 & 12 \\
\hline 34 & $\mathrm{H}$ & - & - & - & 1480 & 13 \\
\hline 35 & $\mathrm{H}$ & - & - & - & 1290 & 4 \\
\hline 36 & $\mathrm{H}$ & - & - & - & 1000 & 2 \\
\hline 37 & $\mathrm{H}$ & - & - & - & 1390 & 2 \\
\hline 38 & $\mathrm{H}$ & - & - & - & 1200 & 3 \\
\hline 39 & $\mathrm{H}$ & - & - & - & 1600 & 1 \\
\hline 40 & $\mathrm{H}$ & - & - & - & 974 & 13 \\
\hline 41 & $\mathrm{H}$ & - & - & - & 1600 & 3 \\
\hline 42 & $\mathrm{H}$ & - & - & - & 460 & 1 \\
\hline 43 & $\mathrm{H}$ & - & - & - & 850 & 3 \\
\hline 44 & $\mathrm{H}$ & - & - & - & 785 & 3 \\
\hline
\end{tabular}

$\beta A P P, \beta$ amyloid precursor protein; DAI, diffuse axonal injury; GFAP, glial fibrillary acid protein; H, hypoxia only; HI, head injury only; HI\&H, head injury and hypoxia. head injury and hypoxic changes, and 12 of 28 cases of hypoxia without history of head injury; 22 of 25 cases who had been ventilated showed positive staining. The majority of cases showed evidence of cerebral swelling.

Conclusions-Axonal bulbs staining positively for $\beta A P P$ may occur in the presence of hypoxia and in the absence of head injury. The role of hypoxia, raised intracranial pressure, oedema, shift effects, and ventilatory support in the formation of axonal bulbs is discussed. The presence of axonal bulbs cannot necessarily be attributed to shearing forces alone.

(F Clin Pathol 1999;52:203-209)

Keywords: $\beta$ amyloid precursor protein; axonal bulbs; diffuse axonal injury; hypoxia

$\beta$ Amyloid precursor protein $(\beta \mathrm{APP})$, is a glycoprotein synthesised within neuronal cytoplasm. ${ }^{1}$ It normally flows along axons in quantities that are undetectable, but accumulates when axonal flow is disturbed. The presence of axonal bulbs has been used as a marker of diffuse axonal injury and, since the early 1980s, to explain the pathophysiology of head injury resulting in loss of consciousness and significant mortality with little or no macroscopic abnormality. ${ }^{2}$ A period of survival of 12 to 24 hours following head trauma is necessary before bulbs can be detected using haematoxylin and eosin (H\&E) or silver stains. However, using immunohistochemical techniques, evidence of flow disturbance and bulb formation may be detected as early as two hours. ${ }^{3}$

Diffuse axonal injury is not the only consequence of non-missile head trauma. It is recognised that there may also be diffuse vascular injury resulting in oedema, hypoxia, and ischaemic brain damage, sometimes associated with swelling and shift effects. ${ }^{4}$ Several investigators have stressed that axonal bulb formation may occur in the absence of a head injury, usually in association with other intracranial pathology. ${ }^{56}$ The possible role of hypoxia in bulb formation has also been raised, but we are not aware of any papers that specifically address this aspect. We now present the results of a study to assess the possible role of hypoxia in the formation of axoplasmic bulbs.

\section{Methods}

The series of cases studied included the following: 28 cases (retrospective and prospective) showing histological evidence of hypoxia in which there was no history of a head injury; four cases in which there was a history of head 
Table 2 Age, sex, cause of death, results of neuropathological examination, survival time, and use of ventilator (V)

\begin{tabular}{|c|c|c|c|c|c|c|}
\hline Case & Sex & Age & Cause of death & Brain histology & Survival time & \\
\hline 1 & M & $62 \mathrm{y}$ & 1a Bronchopneumonia 1b Head injury & DAI & 5.5 weeks & $\mathrm{V}$ \\
\hline 2 & $\mathrm{~F}$ & $59 \mathrm{y}$ & 1a Head injury & DAI & $11 \mathrm{~d}$ & $\mathrm{~V}$ \\
\hline 3 & $\mathrm{~F}$ & $8 \mathrm{y}$ & 1a Diffuse brain injury and subdural haematoma & DAI & $4 \mathrm{~d}$ & $\mathrm{~V}$ \\
\hline 4 & M & $10 \mathrm{y}$ & 1a Head injury $1 \mathrm{~b}$ Road traffic accident & $\mathrm{DAI}$ & $11 \mathrm{~d}$ & $\mathrm{~V}$ \\
\hline 5 & $\mathrm{~F}$ & $18 \mathrm{y}$ & 1a Cerebral contusion and fractured skull & Head injury & $3 \mathrm{~d}$ & $\mathrm{~V}$ \\
\hline 6 & M & $56 \mathrm{y}$ & 1a Fractured skull, cerebral contusion, and subdural haematoma & Head injury & $24 \mathrm{~h}$ & $\mathrm{~V}$ \\
\hline 7 & $\mathrm{M}$ & $29 \mathrm{y}$ & 1a. Inhalation of gastric contents $1 \mathrm{~b}$ Blunt trauma to head 2 Intoxication due to alcohol & Head injury & Minutes & - \\
\hline 8 & $\mathrm{~F}$ & $78 \mathrm{y}$ & 1a Chronic subdural haematoma & Subdural haematoma & $5 \mathrm{~d}$ & $\mathrm{~V}$ \\
\hline 9 & $\mathrm{~F}$ & $47 \mathrm{y}$ & 1a Mechanical asphyxia & $\mathrm{HI}$ and hypoxia & $2 \mathrm{~d}$ & $\mathrm{~V}$ \\
\hline 10 & $\mathrm{M}$ & $26 \mathrm{y}$ & 1a Hypoxic brain damage & $\mathrm{HI}$ and hypoxia & $5 \mathrm{~d}$ & $\mathrm{~V}$ \\
\hline 11 & $\mathrm{M}$ & $20 \mathrm{~m}$ & 1a Swelling of brain and subdural haemorrhage $1 \mathrm{~b}$ Blunt head injury & $\mathrm{HI}$ and hypoxia & $24 \mathrm{~h}$ & $\mathrm{~V}$ \\
\hline 12 & M & $37 \mathrm{y}$ & 1a Head injury & $\mathrm{HI}$ and hypoxia & $24 \mathrm{~h}$ & $\mathrm{~V}$ \\
\hline 13 & M & $30 \mathrm{y}$ & 1a Multiple injuries $1 \mathrm{~b}$ Road traffic accident & $\mathrm{HI}$ and hypoxia & Minutes & - \\
\hline 14 & $\mathrm{~F}$ & $12 \mathrm{~d}$ & 1a Not ascertained (?shaking) & HI and hypoxia & $24 \mathrm{~h}$ & $\mathrm{~V}$ \\
\hline 15 & $\mathrm{M}$ & $73 \mathrm{y}$ & 1a PE and bronchopneumonia $1 \mathrm{~b}$ Subdural haematoma and fractured skull & HI and hypoxia & $19 \mathrm{~d}$ & - \\
\hline 16 & $\mathrm{~F}$ & $38 \mathrm{y}$ & 1a Inhalation of vomit $1 \mathrm{~b}$ Blunt trauma to head & $\mathrm{HI}$ and hypoxia & $4 \mathrm{~h}$ & - \\
\hline 17 & M & $22 \mathrm{y}$ & 1a Stab wounds to neck and leg & Hypoxia & $5 \mathrm{~d}$ & $\mathrm{~V}$ \\
\hline 18 & M & $6 \mathrm{~m}$ & 1a Hypoxic brain damage $1 \mathrm{~b}$ Convulsions of undetermined cause & Hypoxia & $68 \mathrm{~d}$ & $\mathrm{~V}$ \\
\hline 19 & M & $19 \mathrm{y}$ & 1a Bronchopneumonia 1b Hypoxic brain damage 1c Overdose of morphine & Hypoxia & $15 \mathrm{~d}$ & $\mathrm{~V}$ \\
\hline 20 & $\mathrm{~F}$ & $21 \mathrm{y}$ & 1a Hypoglycaemic coma & Hypoxia & $10 \mathrm{~d}$ & - \\
\hline 21 & $\mathrm{~F}$ & $63 \mathrm{y}$ & 1a Failure of vital medullary centres $1 \mathrm{~b}$ Cerebral hypoxia 1c Epilepsy & Hypoxia & $3 \mathrm{~d}$ & $\mathrm{~V}$ \\
\hline 22 & M & $34 \mathrm{y}$ & 1a Hypoxic brain damage and liver necrosis & Hypoxia & $36 \mathrm{~h}$ & $\mathrm{~V}$ \\
\hline 23 & $\mathrm{~F}$ & $22 \mathrm{y}$ & 1a Hypoxic brain damage $1 \mathrm{~b}$ Heroin, alcohol, and nitrazepam poisoning & Hypoxia & $6 \mathrm{~d}$ & $\mathrm{~V}$ \\
\hline 24 & $\mathrm{M}$ & $33 \mathrm{~d}$ & 1a Overlaying & Hypoxia & Hours & - \\
\hline 25 & $\mathrm{~F}$ & $36 \mathrm{y}$ & $\begin{array}{l}\text { 1a Cerebral infarct } 1 \mathrm{~b} \text { Thrombotic occlusion of the right internal carotid artery } 1 \mathrm{c} \\
\text { Operation for removal of malignant peripheral nerve sheath tumour }\end{array}$ & Hypoxia & $3 \mathrm{~d}$ & $\mathrm{~V}$ \\
\hline 26 & M & $10 \mathrm{y}$ & 1a Hypoxic brain damage $1 \mathrm{~b}$ Hanging & Hypoxia & $11 \mathrm{~d}$ & $\mathrm{~V}$ \\
\hline 27 & M & $47 \mathrm{y}$ & 1a unascertained & Hypoxia & $13 \mathrm{~d}$ & $\mathrm{~V}$ \\
\hline 28 & $\mathrm{~F}$ & $2 \mathrm{y}$ & 1a Hypoxic brain damage 1 b Cardiorespiratory arrest $1 \mathrm{c}$ Presumed epileptic seizure & Hypoxia & $3 \mathrm{~d}$ & $\mathrm{~V}$ \\
\hline 29 & M & $10 \mathrm{~m}$ & 1a Sudden Infant Death Syndrome & Hypoxia & Hours & - \\
\hline 30 & M & $19 \mathrm{y}$ & 1a Hepatic encephalopathy $1 \mathrm{~b}$ Acute hepatitis & Hypoxia & $4 \mathrm{~d}$ & $\mathrm{~V}$ \\
\hline 31 & M & $33 \mathrm{y}$ & 1a Asphyxia 1b Plastic bag over head & Hypoxia & Hours & - \\
\hline 32 & $\mathrm{M}$ & $48 \mathrm{y}$ & 1a Epilepsy & Hypoxia & Unknown & - \\
\hline 33 & $\mathrm{~F}$ & $7 \mathrm{~m}$ & 1a Bronchopneumonia $1 \mathrm{~b}$ Infantile spasms, cerebral atrophy and cleft palate & Hypoxia & Hours & - \\
\hline 34 & M & $3 \mathrm{y}$ & 1a Drowning 1b Febrile convulsion & Hypoxia & Minutes & - \\
\hline 35 & $\mathrm{~F}$ & $50 \mathrm{y}$ & 1a Bronchopneumonia $1 \mathrm{~b}$ Status epilepticus & Hypoxia & 25 days & $\mathrm{V}$ \\
\hline 36 & M & $7 \mathrm{~m}$ & 1a Not ascertained & Hypoxia & Hours & - \\
\hline 37 & $\mathrm{~F}$ & $35 \mathrm{y}$ & 1a Epilepsy & Hypoxia & Unknown & - \\
\hline 38 & $\mathrm{~F}$ & $27 \mathrm{y}$ & 1a Hypoxic brain damage $1 \mathrm{~b}$ Poisoning due to heroin and temazepam & Hypoxia & $8 \mathrm{~h}$ & - \\
\hline 39 & M & $31 \mathrm{y}$ & 1a Cerebral hypoxia 1b Epilepsy & Hypoxia & $15 \mathrm{~h}$ & - \\
\hline 40 & $M$ & $5 \mathrm{~m}$ & 1a Overlaying & Hypoxia & $4 \mathrm{~h}$ & - \\
\hline 41 & $\mathrm{~F}$ & $33 \mathrm{y}$ & 1a Heroin and temazepam overdose & Hypoxia & Unknown & - \\
\hline 42 & $\mathrm{~F}$ & $6 \mathrm{~m}$ & $\begin{array}{l}\text { 1a Cerebral hypoxic ischaemic damage } 1 \mathrm{~b} \text { Cardiac arrest during hospital procedure } 2 \\
\text { Multiple congenital abnormalities }\end{array}$ & Hypoxia & $16 \mathrm{~d}$ & $\mathrm{~V}$ \\
\hline 43 & M & $4 \mathrm{~m}$ & 1a Unascertained & Hypoxia & Hours & - \\
\hline 44 & M & $29 \mathrm{w}$ & 1a Diffuse brain damage & Hypoxia & Hours & - \\
\hline
\end{tabular}

DAI, diffuse axonal injury; HI, head injury; m, months; PE, pulmonary thromboembolus; w, weeks; y, years.

trauma but no histological evidence of hypoxia and which the term diffuse axonal injury had not been used in the necropsy report; and eight cases in which there was a history of a head injury but changes of hypoxia were also present. Four cases in which the term "diffuse axonal injury" had been used in the neuropathological report following extensive sampling (at least eight blocks per case) were used as controls for the method, as the original examination using silver stains and immunohistochemistry for $\beta A P P$ had shown the presence of axonal bulbs. As there is no reliable grading system for hypoxic injury to the brain, we did not attempt to grade the changes; there is considerable variation from case to case and relevant changes occur over a short period of time.

The brains had been fixed in $10 \%$ buffered formalin for at least four weeks. Blocks were selected from each case from areas previously described as being particularly vulnerable to axonal bulb formation. ${ }^{78}$ The number of blocks used in each case ranged from one to 19 with an average of four (table 1). These included internal capsule, corpus callosum, superior cerebellar peduncle, and brain stem. The tissue was processed to paraffin using a Shandon Hypercentre XP processing machine. Sections were cut $(7 \mu \mathrm{m})$ and stained with
$\mathrm{H} \& \mathrm{E}$, and others were mounted on aminopropytriethoxysilane coated slides. The latter were subjected to a microwave antigen retrieval procedure using citrate buffer, $\mathrm{pH}$ 6, for 10 minutes in a Proline Microchef microwave. ${ }^{9}$ Following microwave retrieval, slides were washed in Tris buffered saline, $\mathrm{pH}$ 7.6, containing $0.1 \%$ Tween 20 and stained immunohistochemically using a Dako catalysed signal amplification system, horseradish peroxidase K1500. Monoclonal antibodies used with the kit were to $\beta A P P$ (clone $22 \mathrm{c} 11$ Boehringer Mannheim) diluted 1:400, glial fibrillary acid protein (GFAP, Dako) at 1:1000, and CD68-PGM1 (CD68, Dako) at 1:1000. In addition $\beta A P P$ (NCL-B-AMY, Europath) and $\beta A P P$ (Dako), both at 1:50, were used on the control cases. Sections were then dehydrated, cleared, and mounted.

All sections were independently examined "blind" to the original diagnosis by two of us (GNR and WRT). As the present grading system for diffuse axonal injury in part takes into account the presence of tear haemorrhages, which were absent from the cases of hypoxia only, this grading system was not used. Positive staining was graded mild, moderate, and severe, mild representing occasional focal axonal varicosities and severe representing diffuse well formed axonal staining with abundant 

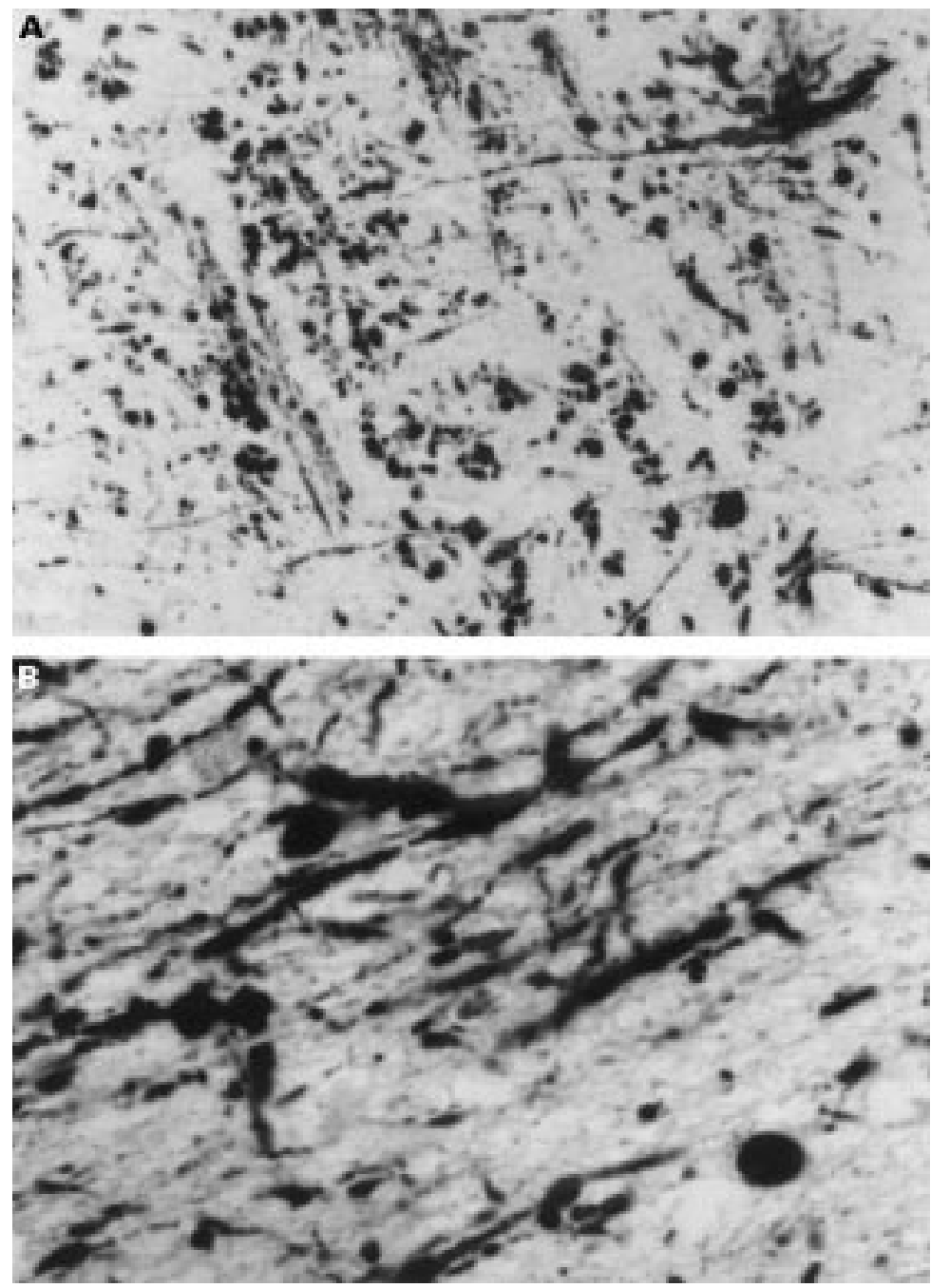

Figure 1 (A) $\beta$ Amyloid precursor positive axonal bulbs and varicosities in a case (No 17) showing neuronal hypoxic change in the absence of a head injury. (B) Higher power view. of cerebral swelling. All showed mild to severe positive staining for $\beta$ APP. The survival time ranged from a few minutes to five days, and three of the four cases had been ventilated. Case 7 showed positivity for $\beta$ APP despite only surviving a few minutes. He had a history of repeated falls and had had a fall downstairs 24 hours before death during which he had sustained a head injury.

Eight cases had had an episode of head trauma and showed histological evidence of hypoxic neuronal damage. Seven of these cases showed mild to moderate staining for $\beta A P P$. Five of these had been ventilated, the survival time varying from 24 hours to five days. One had died after a road traffic accident following which he had been intubated and resuscitated for a short time. The negative case had not been ventilated and had survived four hours. The majority of this group showed an increase in brain weight.

Of 28 cases showing changes characteristic of hypoxia in the absence of a head injury, 12 $(43 \%)$ showed variable positive staining for $\beta A P P$ (table 1, fig 1). In those staining positively for $\beta A P P$, all but one case (No 22) showed increased activity of GFAP and CD68 in astrocytes and microglial cells, respectively. The survival time was known in 25 of these cases and ranged from a few minutes to 68 days. In those showing positive staining the survival time ranged from a few hours to 68 days. Ten of the 12 cases had been ventilated and all showed an increase in brain weight.

Two types of positive staining for $\beta A P P$ was observed in a similar anatomical distribution in all four groups (fig 2). In all blocks examined there was an increase in the intensity of staining of the cytoplasm of neurones. This cytoplasmic staining was observed in some cases in the grey matter of the frontal and parietal lobes in the absence of axonal staining. Positive axonal staining was recorded in the regions of the corpus callosum, lentiform and caudate nuclei, thalamus and subthalamic regions, medulla, and pons. Positive staining within these regions was observed in all blocks from these sites in the cases showing only evidence of hypoxia. Positive axonal staining was also observed in the hippocampus and crus fornicis in two cases of hypoxia alone (fig 3).

The case histories of those cases showing only hypoxic changes and positive staining for $\beta A P P$ are summarised below.

\section{CASE 17}

A 22 year old male was stabbed in the neck and leg, as a result of which he bled profusely. He was taken to hospital where he had an asystolic cardiac arrest for which resuscitation was successful. Following surgery he was considered to have suffered hypoxic brain damage. $\mathrm{He}$ died five days after the injuries.

\section{CASE 18}

A six month old male was admitted unconscious to hospital following severe generalised convulsions. He was managed with ventilatory support but died 68 days after admission. No cause for the convulsions was identified. 
B
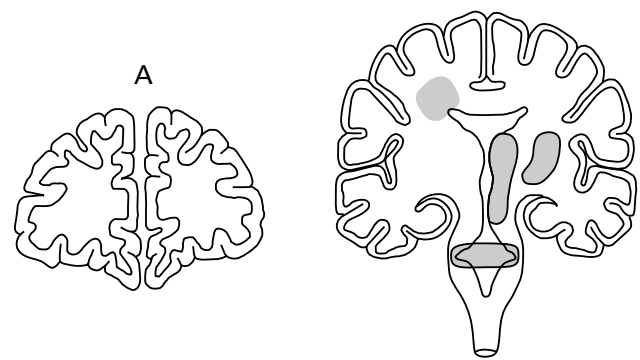

Diffuse axonal injury group

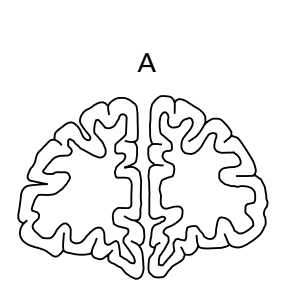

$B$

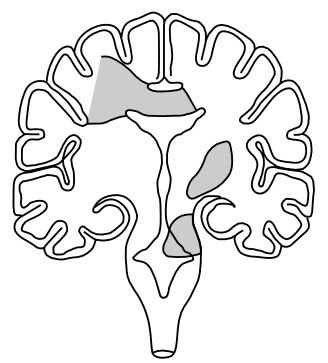

Head injury only

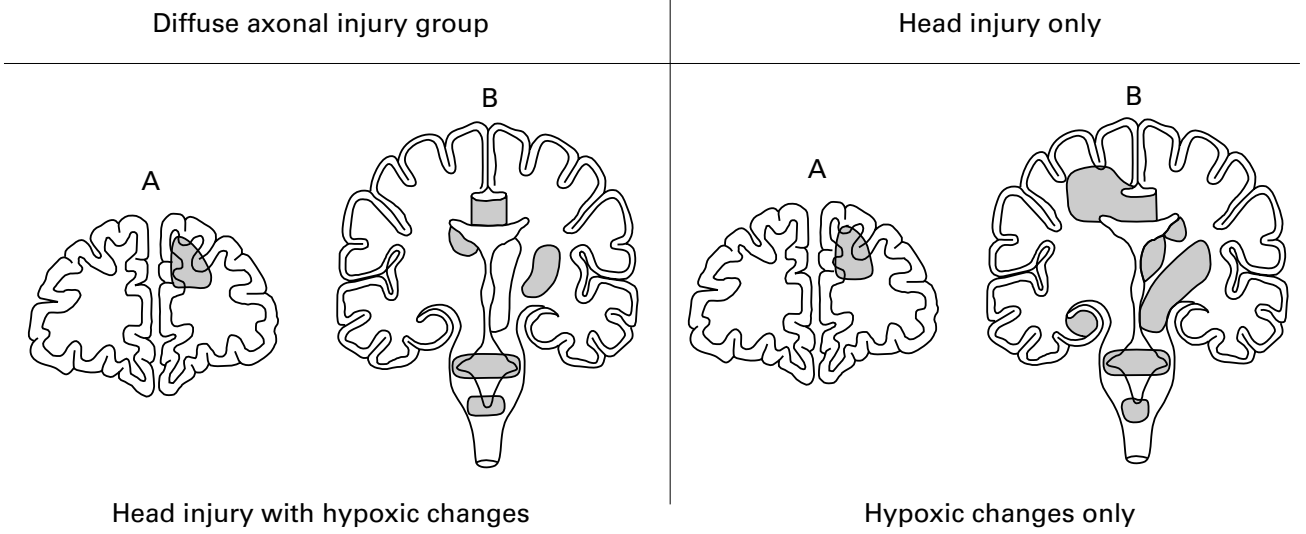

Figure 2 Diagrammatic representation of the anatomical distribution of axonal positivity for $\beta$ amyloid precursor protein in the four study groups.

CASE 19

A 19 year old male was found collapsed at a scene frequented by drug abusers. He was conveyed to hospital where he was diagnosed as having hypoxic brain damage. He developed convulsions in hospital which were treated with diazimuls. He died 15 days after admission. Toxicological testing revealed that he had taken morphine or heroin.

CASE 20

A 21 year old female was admitted to hospital in hypoglycaemic coma. She failed to regain consciousness and died 10 days after admission.

CASE 21

A 63 year old female complained of feeling unwell shortly before collapsing. There was no head injury. She was taken to hospital and placed on a ventilator. She died three days after admission.

CASE 22

A 34 year old male had been paraplegic following a diving accident three years previously. $\mathrm{He}$ ingested a quantity of Khat and LSD; 20 minutes later he became restless with spasms, rapid pulse, and pyrexia. He convulsed and was taken to hospital where he became deeply unconscious. He was ventilated and developed liver failure. He died 36 hours after admission.

CASE 23

A 22 year old female drug misuser collapsed while detained in police custody. There was no head injury. She became deeply unconscious and was conveyed to hospital where hypoxic brain damage was diagnosed. She was venti- lated but died six days after admission. Toxicological testing revealed that she had taken heroin, alcohol, and nitrazepam.

CASE 24

A 33 day old male was fed and taken to bed where he was laid on top of his father's chest. His mother was also in the bed. Both parents had been drinking alcohol. The mother awoke and found the child lying face down, dead, underneath the father.

CASE 25

A 36 year old female with neurofibromatosis underwent surgical removal of a right sided neck tumour. During the operation she bleed excessively, requiring 5 units of blood. Postoperatively she was found to have suffered right sided cerebral infarction. She was ventilated but died three days postoperatively.

\section{CASE 26}

A 10 year old male was found hanging by a dog lead from a railing. Resuscitation was successful at the scene. He was conveyed to hospital where hypoxic brain damage was diagnosed. $\mathrm{He}$ was ventilated but died 11 days after admission.

CASE 27

A 47 year old male was found collapsed, outdoors, face down in mud. He was conveyed to hospital where respiration was recorded as been spontaneous. He was intubated and treated on the intensive care unit for 13 days before he died.

\section{CASE 28}

A two year old female with a history of febrile convulsions and operative correction of an 

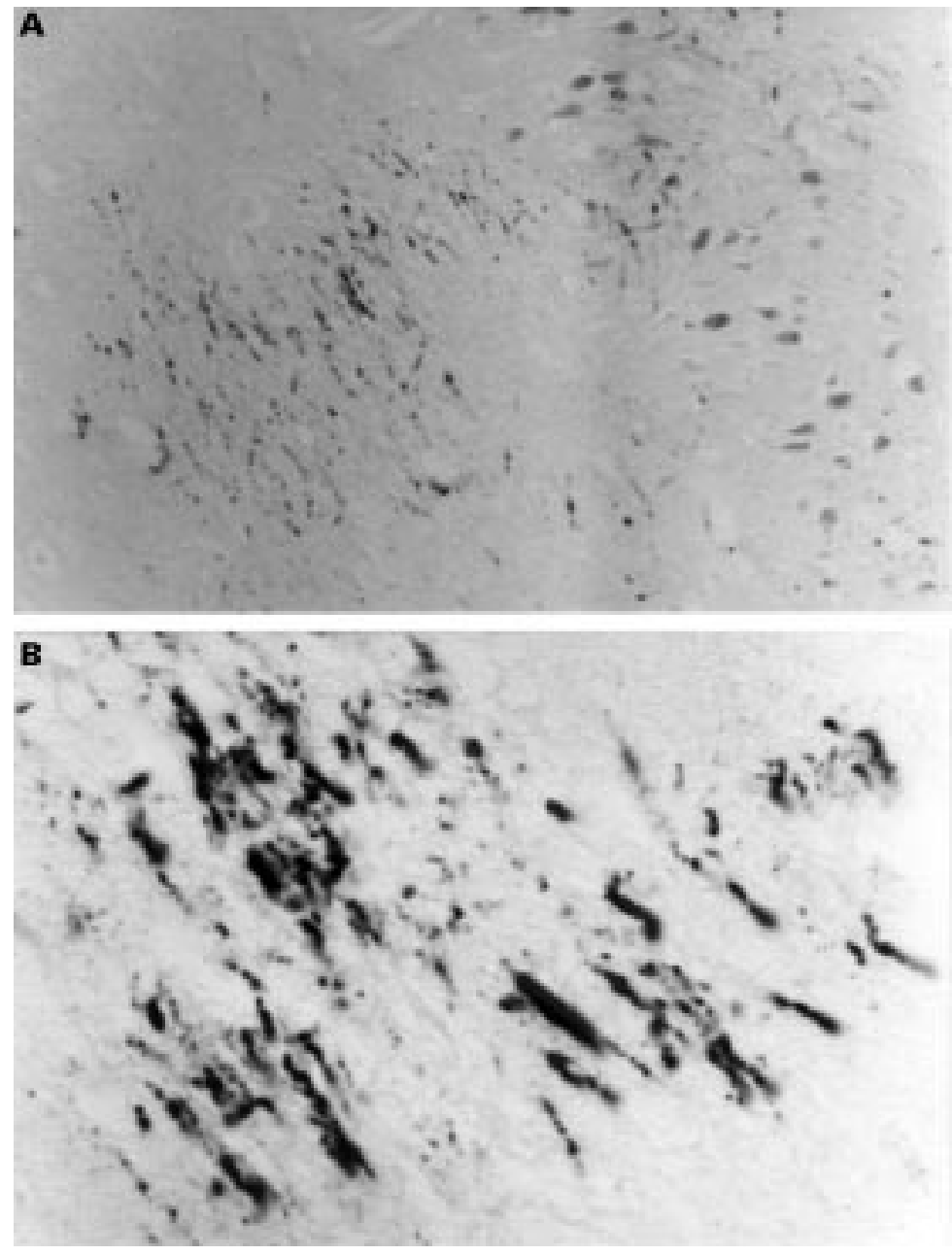

Figure 3 (A) $\beta$ amyloid precursor protein positivity within the hippocampus of a case with hypoxia only. (B) High power view.

intussusception was admitted to hospital following an asystolic cardiac arrest lasting at least 40 minutes. Resuscitation was successful but despite intensive care support, she died three days after admission.

\section{Discussion}

The term diffuse axonal injury was first used in the early 1980 s to explain the pathophysiology of non-missile head injury resulting in loss of consciousness, persistent vegetative state, and significant mortality often in the absence of macroscopic evidence of brain injury. ${ }^{10} 11$ The presence of axonal retraction bulbs is often used as evidence of diffuse axonal injury. These may be demonstrated by H\&E or silver stains after a survival period of 12 to 24 hours, and with $\beta$ APP immunohistochemistry axonal debris may occasionally be detected one year after head injury. ${ }^{12}$ Sensitivity can be increased to two to three hours by immunohistochemical techniques, particularly using an antibody to $\beta A P P{ }^{5}$

Most investigators have concentrated on the detection and timing of axonal bulbs following head trauma, emphasising the role of shearing forces generated during the injury resulting in breakdown of the integrity of axolemmal membrane and calcium influx mediated ul- trastructural collapse. ${ }^{13-15}$ The role of hypoxia in the formation of axonal bulbs has received little attention. Sherriff et al pointed out that $\beta A P P$ is not a true diagnostic marker for head injury but rather a marker of more generalised damage to the brain, although in their series only one case showed $\beta A P P$ staining as a result of hypoxia alone. ${ }^{5}$ Jellinger maintained that diffuse axonal injury was a secondary event following hypoxic brain damage, posttraumatic oedema, or secondary compression of the brainstem as a result of tentorial herniation, ${ }^{16}{ }^{17}$ although other workers have dismissed this idea. ${ }^{18}$ Subsequently the possibility that there may be other pathophysiological explanations, including the effects of hypoxia and ventilatory support, has received little attention.

During non-missile head injury, nondisruptive traumatic axonal damage is thought to occur as a result of shearing forces generated during the injury. The effects of this are greatest at the weakest part of the axon, namely the node of Ranvier. Microtubular and cytoskeletal disruption leads to reduction of axonal flow, with accumulation of axonal proteins at the point of swelling that develops at the site of damage, resulting in the formation of the axonal bulb.

Diffuse axonal injury is classified into three grades, which take into account the site of formation of the bulbs. In grade 1 there is widespread axonal damage in the corpus callosum, white matter of the cerebral hemispheres, and brain stem; in grade 2 there are additional focal abnormalities in the corpus callosum with small "tear" haemorrhages; and in grade 3 there are severe axonal abnormalities including the involvement of the rostral brain stem. ${ }^{19}$ Extensive tissue sampling is recommended to enable diagnosis and grading. ${ }^{12}$

Although diffuse axonal injury is regarded as a consequence of head injury, several investigators have drawn attention to the fact that $\beta$ APP staining occurs in unrelated conditions. Sherriff et al reported that five of 13 control cases in their series had positive staining for $\beta A P P .^{5}$ Of these, four cases had non-traumatic cerebral pathology listed as cerebral infarction, Binswanger's disease, intracranial haemorrhage, and metastatic melanoma with haemorrhage. The fifth case died of congestive cardiac failure with $\beta A P P$ staining attributed to the effects of hypoxia. Although no brain weights were quoted, the survival times were recorded as from four to over 200 hours. There was indication as to whether the cases had required ventilatory support.

Gentleman et al also reported that 10 of their 23 controls with no evidence of head injury showed positive staining, but all of their cases had other cerebral abnormalities including infarction, abscess, hypoxia, and multiple sclerosis. ${ }^{6}$ Geddes draws attention to these results and emphasises other non-traumatic causes, particularly infarction, and recommends caution in the interpretation of a positive staining in the early cases. ${ }^{12}$ Case 7 in our series raises the possibility that a positive reaction may be unrelated to the most recent 
cerebral insult and may represent bulb formation from an earlier episode of trauma.

The results presented here show that $\beta A P P$ staining may occur in the presence of hypoxia alone and in a distribution similar to that described in diffuse axonal injury. None of the 12 cases studied (cases 17 to 28 ) had suffered a head injury, and 10 of them had histological evidence of hypoxic brain damage as a result of non-cerebral events. In all 12 cases the brain weight was increased and 10 had been ventilated for hours to days before death. Of the remaining 16 cases showing evidence of hypoxia but a negative reaction for $\beta A P P$, only three had been ventilated. The majority showed an increase in brain weight. In seven of these the survival time was only a few hours and therefore there may have been insufficient time for the development of axonal bulbs. Of note is the fact that all four cases originally described as showing diffuse axonal injury, three of the four head injury cases with no hypoxic changes, and five of the eight cases of mixed head injury/hypoxia had been ventilated, and the majority showed an increase in brain weight.

Cerebral lesions similar to diffuse axonal injury have been reported in primates without evidence of hypoxia or raised intracranial pressure..$^{20}$ Diffuse axonal injury is considered to be a primary post-traumatic lesion with cerebral oedema, raised intracranial pressure, and hypoxic and ischaemic brain damage occurring as secondary events. ${ }^{21} 22$ The results presented in this paper, however, show that similar changes may occur as a result of hypoxia in the absence of a head injury.

Hypoxia is a generic term used to indicate a disruption in the delivery and utilisation of oxygen for cellular respiration. It may be classified into several types depending on the underlying cause. Hypoxic brain damage is estimated to occur in $91 \%$ of all head injuries. ${ }^{23}$ Our study includes examples of anaemic and stagnant hypoxia and hypoglycaemic brain damage with positive staining for $\beta$ APP. It seems likely, therefore, that axonal bulbs can be the result of hypoxia and associated metabolic changes rather than purely the result of shearing forces. Metabolic disturbances, loss of cellular membrane integrity, calcium influx, and ultrastructural changes are not unique to shearing injuries and are a feature of cell injury in other tissues. Baiden-Amissah et al have proposed that increased production of $\beta A P P$ is induced during hypoxic cerebral injury and that this induction is part of a more generalised cerebral acute phase response to hypoxic injury. ${ }^{24}$ This could account for the increase in $\beta \mathrm{APP}$ expression within the cytoplasm of neurones following cerebral insult.

The possibility that the "respirator brain," the extreme end of stagnant hypoxia, may also be associated with similar consequences must also be considered. ${ }^{19}$ So far few investigators have commented upon whether any of their reported cases of diffuse axonal injury had required ventilatory support, which is a common requirement in severe head injuries or intracranial haemorrhage. Sherriff et al re- corded that nine of their 36 cases had been ventilated and that all five cases of head injury, and two of the four cases with no evidence of a head injury, showed positive staining. ${ }^{5}$ Overall, 25 of our cases had been ventilated and 22 of these showed positive staining for $\beta$ APP. These included all of the diffuse axonal injury group, all three of the head injury group in which $\beta A P P$ staining was attributed to the admission injury, five of the eight with head injury and hypoxia, 10 of 12 staining positively in the hypoxia alone group, and three of the cases staining negatively in the hypoxia alone group. These figures suggest that ventilatory support may be an important factor, although the absence of staining in three cases of hypoxia alone indicates that ventilatory support is not essential.

The role of raised intracranial pressure and cerebral oedema, as reflected by an increase in brain weight, has not been addressed since Jellinger's reports. ${ }^{16}{ }^{17}$ Raised intracranial pressure and unilateral intracranial haemorrhage may result in shift effects associated with stretching of the axons at sites reported to show $\beta A P P$. This may affect axoplasmic flow and cellular structure, resulting in the formation of axoplasmic bulbs.

Our study shows the need for further assessment of the role of hypoxia, raised intracranial pressure, ventilatory support, and shift effects in the formation of axonal bulbs.

We wish to thank Professor M A Green and Drs J Clark, C Milroy, N Carter, and C Smith for allowing the use of their cases. We also wish to thank the laboratory staff of the Departments of Forensic Pathology and Neuropatholgy for technical advice and block retrieval, Renata Green for retrieving the case histories, Dako UK and Europath Ltd for providing samples of their $\beta A P P$ antibodies, and Mr Ian Mckarill, formally of Dako, for technical advice regarding the Dako CSA K1500 kit.

1 Selkoe DJ. Normal and abnormal biology of the $\beta$-amyloid precursor protein. Anпu Rev Neurosci 1994;17:489-517.

2 Gennarelli TA. The spectrum of axonal injury. Neuropathol Appl Neurobiol 1996;22:509-13.

3 Sherriff FE, Bridges LR, Gentleman SM, et al. Markers of Sherriff FE, Bridges LR, Gentleman SM, et al. Markers of
axonal injury in post mortem human brain. Acta Neuropathol 1994;88:433-9.

4 Gennarelli TA. Mechanisms of brain injury. 7 Emerg Med 1993;11:5-11.

5 Sherriff FE, Bridges R, Sivalaganathan S. Early detection of axonal injury after human head trauma using immunocytochemistry for $\beta$-amyloid precursor protein. Acta Neuropathol 1994;87:55-62.

6 Gentleman SM, Roberts GW, Gennarelli TA, et al. Axonal njury: a universal consequence of fatal closed head injury? Acta Neuropathol 1995;89:537-43.

7 Graham DI, Gentleman SM, Lynch A, et al. Distribution of $\beta$-amyloid protein in the brain following severe head injury. Neuropathol Appl Neurobiol 1995;21:27-34.

8 Geddes JF, Vowles GH, Beer TW, et al. The diagnosis of diffuse axonal injury: implications for forensic practice. Neuropathol Appl Neurobiol 1997;23:339-47.

9 Sherriff FE, Bridges LR, Jackson P. Microwave antigen retrieval of $\beta$-amyloid precursor protein immunoreactivity. retrieval of $\beta$-amyloid precursor protein inm

10 Adams HJ, Graham DI. Diffuse brain damage in nonmissile head injury. In: Anthony PP, Macsween RNM, eds. Recent advances in histopathology, 12th ed. London: Churchill Livingstone, 1984:241-58.

11 Ironside JW. Blunt injury to the head. In: Mason JK, ed. The pathology of trauma. London: Edward Arnold, 1993:166-7. 2 Geddes JF. What's new in the diagnosis of head injury? $\mathcal{f}$ Clin Pathol 1997;50:271-4

13 Novack TA, Dillon MC, Jackson WT. Neurochemical mechanisms in brain injury and treatment: a review. $\mathcal{F}$ Clin Exp Neuropsychol 1996;18:685-706.

14 Christman CW, Grady M, Walker SA, et al. Ultrastructural studies of diffuse axonal injury in humans. 7 Neurotrauma 1994;11:173-86.

15 Adams JH, Graham DI, Gennarelli TA, et al. Diffuse axonal injury in non-missile head injury. $\mathcal{F}$ Neurol Neurosurg Psychiatry 1991;54:481-3.

16 Jellinger K, Seitelberger F. Protracted post-traumatic encephalopathy. Pathology, pathogenesis and clinical implications. F Neurol Sci 1970;10:51-94. 
17 Jellinger K. Pathology and pathogenesis of the apallic syndromes following closed head injuries. In: Ore GD, Gerstenbrand F, Lucking $\mathrm{CH}$, et al, eds. The appallic syndrome. Berlin: Springer, 1977:88-103.

18 Adams JH, Graham DI, Murray LS, et al. Diffuse axonal injury due to nonmissile head injury in humans: an analysis of 45 cases. Ann Neurol 1982;12:557-63.

19 Graham DI, Lantos PL, eds. Greenfield's neuropathology, 6th ed. London: Arnold, 1997:197-314.

20 Gennarelli TA, Thibault LE, Adams JH, et al. Diffuse axona injury and traumatic coma in the primate. Ann Neurol 1982;12:564-74.
21 Tomei G, Sganzerla E, Spagnoli D, et al. Post traumatic diffuse cerebral lesions. Relationships between clinical course, CT findings and ICP. F Neurosurg Sci 1991;35:61-75.

22 Adams JH, Doyle D, Graham DJ, et al. Gliding contusions in non missile head injury in humans. Arch Pathol Lab Med 1986;110:485-8.

23 McIntyre JP. Hypoxia. Br f Hosp Med 1969;2:1113-29.

24 Baiden-Amissah K, Cox PM, Joashi U, et al. Induction of $\beta$-amyloid precursor protein ( $\beta \mathrm{APP}$ ) expression after neonatal hypoxic ischaemic cerebral injury (HII) [abstr]. $\mathcal{F}$ Pathol 1997;182(suppl):49A. 